\title{
On Multivalued Hybrid Contractions with Applications
}

\author{
Monairah Alansari, ${ }^{1}$ Shehu Shagari Mohammed $\mathbb{D}^{\mathrm{D}},{ }^{2}$ Akbar Azam $\mathbb{D}^{\mathrm{D}},{ }^{3}$ and Nawab Hussain $\mathbb{D D}^{1}$ \\ ${ }^{1}$ Department of Mathematics, King Abdulaziz University, P.O. Box 80203, Jeddah 21589, Saudi Arabia \\ ${ }^{2}$ Department of Mathematics, Faculty of Physical Sciences, Ahmadu Bello University, Nigeria \\ ${ }^{3}$ Department of Mathematics, COMSATS University, Chak Shahzad, Islamabad 44000, Pakistan
}

Correspondence should be addressed to Shehu Shagari Mohammed; shagaris@ymail.com

Received 11 May 2020; Accepted 18 June 2020; Published 11 July 2020

Guest Editor: Antonio Francisco Roldan Lopez de Hierro

Copyright (c) 2020 Monairah Alansari et al. This is an open access article distributed under the Creative Commons Attribution License, which permits unrestricted use, distribution, and reproduction in any medium, provided the original work is properly cited.

\begin{abstract}
Recently, a notion of $b$-hybrid contraction for single-valued mappings in the framework of $b$-metric spaces which unify and improve several significant existing results in the corresponding literature was introduced. This paper presents a multivalued generalization for such contraction. Moreover, one of our obtained results is applied to analyze some solvability conditions of Fredholm-type integral inclusions. Nontrivial examples are also provided to support the assertions of our theorems.
\end{abstract}

\section{Introduction}

The Banach contraction principle is the first most wellknown, simple, and versatile classical result in fixed point theory with metric space structure. More than a handful of literature embraces applications and generalizations of this principle from different perspectives, for example, by weakening the hypotheses, employing different mappings and various forms of metric spaces. In this context, the work of Rhoades [1] is useful for visiting important modifications of Banach-type contractive definitions. In 1969, Nadler [2] gave a generalization of the Banach contraction principle for multivalued contraction mappings by using the Hausdorff metric and established the first fixed point theorem for multivalued mappings defined on metric space. Since then, a number of generalizations in diverse frames of Nadler's fixed point result have been investigated by several authors (see, for example, [3-9] and references therein).

The analysis of new spaces and their properties has been an interesting topic among the mathematical research community. In this direction, the notion of $b$-metric spaces is presently thriving. The idea commenced with the work of Bakhtin [10] and Bourbaki [11]. Thereafter, Czerwik [12] gave a postulate which is weaker than the classical triangle inequality and formally established a $b$-metric space with a view of improving the Banach fixed point theorem. Mean- while, the notion of $b$-metric spaces is gaining enormous generalizations (see, for example, [8, 13-15]). For a recent short survey on basic concepts and results in fixed point theory in the framework of $b$-metric spaces, we refer the interested reader to Karapinar [16]. On similar development, one of the active branches of fixed point theory that is also currently drawing the attentions of researchers is the study of hybrid contractions. The concept has been viewed in two directions; viz., first, hybrid contraction deals with contractions involving both single-valued and multivalued mappings, and the second merges linear and nonlinear contractions. Recently, Karapinar and Fulga [17] introduced a new notion of $b$ hybrid contraction in the frame of $b$-metric space and studied the existence and uniqueness of fixed points for such contraction. Their ideas merged several existing results in the corresponding literature. Interestingly, hybrid fixed point theory has potential applications in functional inclusions, optimization theory, fractal graphics, discrete dynamics for set-valued operators, and other areas of nonlinear functional analysis. For some work on this line, the reader may consult [17-21].

Integral inclusions arise in several problems in mathematical physics, control theory, critical point theory for nonsmooth energy functionals, differential variational inequalities, fuzzy set arithmetic, traffic theory, etc. (see, for instance, [22-24]). Usually, the first most concerned problem in the study of 
integral inclusions is the conditions for existence of its solutions. In this direction, several authors have applied different fixed point approaches and topological methods to obtain existence results of integral inclusions in abstract spaces (see, for example, Appell et al. [22], Cardinali and Papageorgiou [24], Kannan and O'Regan [25], Pathak et al. [9], Sintamarian [26], and the references therein). Most of the results established in the above papers are based on the multivalued analogs of the Banach, Leray-Schauder, Matelli, Schauder, and Sadovskii-type fixed point theorems. In addition, the ambient space of the existence theorems is either a Banach space or classical metric space.

Following the above development, we define in this paper the idea of $b$-hybrid multivalued contraction on a $b$-metric space and analyze conditions for existence of fixed points for such contraction. A nontrivial example which supports the hypotheses of our results is provided. Thereafter, a few significant particular cases are deduced which include the recent results of Karapinar and Fulga [17] and many others. Furthermore, one of our results is applied to investigate sufficient conditions for existence of solutions to an integral inclusion of Fredholm type. The latter concept is adapted from Sintamarian [26]. However, our technique, being obtained through a $b$-hybrid multivalued contraction in the setting of $b$-metric spaces, leads to a new existence principle which extends and complements the existing literature.

\section{Preliminaries}

In this section, we collect some important notations, useful definitions, and basic results coherent with the literature. Throughout this paper, we denote by $\mathbb{N}, \mathbb{R}_{+}$, and $\mathbb{R}$ the sets of natural numbers, nonnegative real numbers, and real numbers, respectively. These preliminary concepts are recorded from $[2,12,17]$.

In 1993, Czerwik [12] introduced the notion of a $b$-metric space as follows.

Definition 1 (see [12]). Let $X$ be a nonempty set and $\eta \geq 1$ be a constant. Suppose that the mapping $\sigma: X \times X \longrightarrow \mathbb{R}_{+}$satisfies the following conditions for all $x, y, z \in X$ :

(i) $\sigma(x, y)=0$ if and only if $x=y$ (self-distancy)

(ii) $\sigma(x, y)=\sigma(y, x)$ (symmetry)

(iii) $\sigma(x, y) \leq \eta[\sigma(x, z)+\sigma(z, y)] \quad$ (weighted triangle inequality)

Then, the tripled $(X, \sigma, \eta)$ is called a $b$-metric space.

It is noteworthy that every metric is a $b$-metric with the parameter $\eta=1$. Also, in general, a $b$-metric is not a continuous functional. Hence, the class of $b$-metric is larger than the class of classical metric.

Example 1 (see [27]). Let $X=l_{p}(\mathbb{R})$ with $0<p<1$, where

$$
l_{p}(\mathbb{R})=\left\{\left\{x_{n}\right\}_{n \in \mathbb{N}} \subseteq \mathbb{R}: \sum_{n=1}^{\infty}\left|x_{n}\right|^{p}<\infty\right\} .
$$

Define $\sigma: X \times X \longrightarrow \mathbb{R}_{+}$as

$$
\sigma(x, y)=\left(\sum_{n=1}^{\infty}\left|x_{n}-y_{n}\right|^{p}\right)^{1 / p},
$$

where $x=\left\{x_{n}\right\}_{n \in \mathbb{N}}$ and $y=\left\{y_{n}\right\}_{n \in \mathbb{N}}$. Then, $\sigma$ is a $b$-metric with parameter $\eta=2^{1 / p}$ and hence, $\left(X, \sigma, 2^{1 / p}\right)$ is a $b$-metric space.

Example 2 (see [28]). Let $X=\mathbb{N} \cup\{\infty\}$ and $\sigma: X \times X \longrightarrow$ $\mathbb{R}_{+}$be defined by

$$
\sigma(x, y)= \begin{cases}0, & \text { if } x=y, \\ \left|\frac{1}{x}-\frac{1}{y}\right|, & \text { if } x, y \text { are even or } x y=\infty \\ 5, & \text { if } x, y \text { are odd and } x \neq y \\ 2, & \text { otherwise. }\end{cases}
$$

Then, $(X, \sigma)$ is a $b$-metric space with parameter $\eta=3$, but $\sigma$ is not a continuous functional.

Definition 2 (see [29]). Let $(X, \sigma, \eta)$ be a $b$-metric space. A sequence $\left\{x_{n}\right\}_{n \in \mathbb{N}}$ is said to be

(i) convergent if and only if there exists $x \in X$ such that $\sigma\left(x_{n}, x\right) \longrightarrow 0$ as $n \longrightarrow \infty$, and we write this as $\lim _{n \rightarrow \infty} \sigma\left(x_{n}, x\right)=0$

(ii) Cauchy if and only if $\sigma\left(x_{n}, x_{m}\right) \longrightarrow 0$ as $n, m \longrightarrow \infty$

(iii) complete if every Cauchy sequence in $X$ is convergent

In a $b$-metric space, the limit of a sequence is not always unique. However, if a $b$-metric is continuous, then every convergent sequence has a unique limit.

Definition 3 (see [29]). Let $(X, \sigma, \eta)$ be a $b$-metric space. Then, a subset $A$ of $X$ is called

(i) compact if and only if for every sequence of elements of $A$, there exists a subsequence that converges to an element of $A$

(ii) closed if and only if for every sequence $\left\{x_{n}\right\}_{n \in \mathbb{N}}$ of elements of $A$ that converges to an element $x$, we have $x \in A$

Definition 4 (see [25]). A nonempty subset $A$ of $X$ is called proximal if, for each $x \in X$, there exists $a \in A$ such that $\sigma(x$, a) $=\sigma(x, A)$.

Throughput this paper, we shall denote by $C B(X), \mathscr{P}^{r}(X)$, $\mathscr{P}_{b}^{r}(X)$, and $\mathscr{K}(X)$, the set of all nonempty closed and bounded subsets of $X$, the family of all nonempty proximal subsets of $X$, the set of all bounded proximal subsets of $X$, and the class of nonempty compact subsets of $X$, respectively. 
Let $(X, \sigma, \eta)$ be a $b$-metric space. For $A, B \in \mathscr{P}^{r}(X)$, the function $H_{b}: \mathscr{P}^{r}(X) \times \mathscr{P}^{r}(X) \longrightarrow \mathbb{R}_{+}$, defined by

$H_{b}(A, B)= \begin{cases}\max \left\{\sup _{x \in A} \sigma(x, B), \sup _{x \in B} \sigma(x, A)\right\}, & \text { if it exists, } \\ \infty, & \text { otherwise }\end{cases}$

is called Hausdorff-Pompeiu $b$-metric on $\mathscr{P}^{r}(X)$ induced by the $b$-metric $\sigma$, where

$$
\sigma(x, A)=\inf _{y \in A} \sigma(x, y) .
$$

Definition 5. Let $(X, \sigma, \eta)$ be a metric space, and $\mathcal{N}(X)$ denotes the family of nonempty subsets of $X$. A set-valued mapping $T: X \longrightarrow \mathcal{N}(X)$ is called a multivalued map. A point $u \in X$ is said to be a fixed point of $T$ if $u \in T u$.

Remark 6. Since every compact set is proximal and every proximal set is closed (see [25]), we have the inclusions

$$
\mathscr{K}(X) \subseteq \mathscr{P}^{r}(X) \subseteq C B(X) \subseteq \mathscr{N}(X) .
$$

Definition 7 (see $[17,30]$ ). A nondecreasing function $\varphi$ : $\mathbb{R}_{+} \longrightarrow \mathbb{R}_{+}$is called

(i) a $c$-comparison function if $\varphi^{n}(t) \longrightarrow 0$ as $n \longrightarrow \infty$ for every $t \in \mathbb{R}_{+}$

(ii) a $b$-comparison function if there exist $k_{0} \in \mathbb{N}, \lambda \in(0$, 1) and a convergent nonnegative series $\sum_{n=1}^{\infty} x_{n}$ such that $\eta^{k+1} \varphi^{k+1}(t) \leq \lambda \eta^{k} \varphi^{k}(t)+x_{k}$, for $\eta \geq 1, k \geq k_{0}$ and any $t \geq 0$, where $\varphi^{n}$ denotes the $n^{\text {th }}$ iterate of $\varphi$

Denote by $\Omega$ the family of functions $\varphi: \mathbb{R}_{+} \longrightarrow \mathbb{R}_{+}$satisfying the following conditions:

(i) $\varphi$ is a $b$-comparison function

(ii) $\varphi(t)=0$ if and only if $t=0$

(iii) $\varphi$ is continuous

Remark 8 (see [17]). A $b$-comparison function is a $c$-comparison function when $\eta=1$. Moreover, it can be shown that a $c$-comparison function is a comparison function, but the converse is not always true. For further properties of comparison function, see [31].

Lemma 9 (see [30]). For a comparison function $\varphi: \mathbb{R}_{+} \longrightarrow$ $\mathbb{R}_{+}$, the following properties hold:

(i) Each iterate $\varphi^{n}, n \in \mathbb{N}$, is also a comparison function

(ii) $\varphi(t)<t$ for all $t>0$

Lemma 10 (see [30]). Let $\varphi: \mathbb{R}_{+} \longrightarrow \mathbb{R}_{+}$be a b-comparison function. Then, the series $\sum_{k=0}^{\infty} \eta^{k} \varphi^{k}(t)$ converges for every $t \in \mathbb{R}_{+}$.

Remark 11 (see [17]). In Lemma 10, every $b$-comparison function is a comparison function and thus, in Lemma 9, every $b$-comparison function satisfies $\varphi(t)<t$.

Lemma 12 (see [32]). Let $(X, \sigma, \eta)$ be a b-metric space. For $A, B \in \mathscr{K}(X)$ and $x, y \in X$, the following conditions hold:

(i) $\sigma(x, B) \leq H_{b}(A, B)$, for any $x \in A$

(ii) $\sigma(x, A) \leq \eta[\sigma(x, y)+\sigma(y, A)]$

(iii) $\sigma(x, A)=0 \Leftrightarrow x \in A$

(iv) $H_{b}(A, B)=0 \Leftrightarrow A=B$

(v) $H_{b}(A, B)=H(B, A)$

(vi) $H_{b}(A, B) \leq \eta\left[H_{b}(A, C)+H_{b}(C, B)\right]$

\section{Main Results}

We start this section by inaugurating the following definition of $b$-hybrid multivalued contraction.

Definition 13. Let $(X, \sigma, \eta)$ be a $b$-metric space and $S, T$ : $X \longrightarrow \mathscr{P}^{r}(X)$ be multivalued maps. Then, the pair $(S, T)$ is said to form a $b$-hybrid multivalued contraction, if for all $x$, $y \in X$, we have

$$
H_{b}(S x, T y) \leq \varphi\left(\mathscr{C}_{(S, T)}^{r}(x, y)\right)
$$

where $\varphi \in \Omega, r \geq 0$, and $a_{i} \geq 0, i=1,2,3,4$ with $\sum_{i=1}^{4} a_{i}=1$ and

$$
\mathscr{C}_{(S, T)}^{r}(x, y)=\left\{\begin{array}{l}
{\left[a_{1}(\sigma(x, y))^{r}+a_{2}\left(\sigma(x, S x)^{r}+a_{3}\left(\sigma(y, T y)^{r}+a_{4}\left(\frac{\sigma(y, S x)+\sigma(x, T y)}{2 \eta}\right)^{r}\right]^{1 / r}, \quad \text { for } r>0, x, y \in X,\right.\right.} \\
(\sigma(x, y))^{a_{1}}(\sigma(x, S x))^{a_{2}}(\sigma(y, T y))^{a_{3}}\left(\frac{\sigma(x, T y)+\sigma(y, S x)}{2 \eta}\right)^{a_{4}}, \quad \text { for } r=0, x, y \in X \backslash \mathscr{F}_{i x}(S, T),
\end{array}\right.
$$


where

$$
\mathscr{F}_{i x}(S, T)=\{x, y \in X: x \in S x, y \in T y\} .
$$

Our main result runs as follows.

Theorem 14. Let $(X, \sigma, \eta)$ be a complete b-metric space and $S, T: X \longrightarrow \mathscr{P}^{r}(X)$ be multivalued maps. Suppose that for each $x, y \in X, S x$ and Ty are nonempty bounded proximal subsets of $X$. If the pair $(S, T)$ forms a b-hybrid multivalued contraction, then $S$ and $T$ have a common fixed point in $X$.

Proof. Let $x_{0} \in X$, then, by hypotheses, $S x_{0} \in \mathscr{P}_{b}^{r}(X)$. Choose $x_{1} \in S x_{0}$ such that $\sigma\left(x_{0}, x_{1}\right)=\sigma\left(x_{0}, S x_{0}\right)$. Similarly, $T x_{1} \in \mathscr{P}_{b}^{r}$ $(X)$, by assumption. So, we can find $x_{2} \in T x_{1}$ such that by proximality of $T, \sigma\left(x_{1}, x_{2}\right)=\sigma\left(x_{1}, T x_{1}\right)$. Continuing in this fashion, we generate a sequence $\left\{x_{n}\right\}_{n \in \mathbb{N}}$ of elements of $X$ such that

$$
\begin{aligned}
x_{2 p+1} & \in S x_{2 p}, \\
x_{2 p+2} & \in T x_{2 p+1} \\
\sigma\left(x_{2 p}, x_{2 p+1}\right) & =\sigma\left(x_{2 p}, S x_{2 p}\right), \\
\sigma\left(x_{2 p+1}, x_{2 p+2}\right) & =\sigma\left(x_{2 p+1}, T x_{2 p+1}\right), \quad p \in \mathbb{N} .
\end{aligned}
$$

By Lemma 12 and the above relations, we obtain

$$
\begin{aligned}
\sigma\left(x_{2 p}, x_{2 p+1}\right) & \leq H_{b}\left(S x_{2 p}, T x_{2 p-1}\right), \\
\sigma\left(x_{2 p+1}, x_{2 p+2}\right) & \leq H_{b}\left(S x_{2 p}, T x_{2 p+1}\right) .
\end{aligned}
$$

Suppose that $x_{2 p}=x_{2 p+1}$, for some $p \in \mathbb{N}$ and $r>0$. Then, from (9), we have

$$
\begin{aligned}
\mathscr{C}_{(S, T)}^{r} & \left(x_{2 p}, x_{2 p+1}\right) \\
= & {\left[a_{1}\left(\sigma\left(x_{2 p}, x_{2 p+1}\right)\right)^{r}+a_{2}\left(\sigma\left(x_{2 p}, S x_{2 p}\right)^{r}\right.\right.} \\
& +a_{3}\left(\sigma\left(x_{2 p+1}, T x_{2 p+1}\right)^{r}\right. \\
& \left.+a_{4}\left(\frac{\sigma\left(x_{2 p+1}, S x_{2 p}\right)+\sigma\left(x_{2 p}, T x_{2 p+1}\right)}{2 \eta}\right)^{r}\right]^{1 / r}
\end{aligned}
$$

$$
\begin{aligned}
= & {\left[a_{1}\left(\sigma\left(x_{2 p}, x_{2 p+1}\right)\right)^{r}+a_{2}\left(\sigma\left(x_{2 p}, x_{2 p+1}\right)\right)^{r}\right.} \\
& +a_{3}\left(\sigma\left(x_{2 p+1}, x_{2 p+2}\right)\right)^{r} \\
& \left.\left.+a_{4}\left(\frac{\sigma\left(x_{2 p+1}, x_{2 p+1}\right)+\sigma\left(x_{2 p}, x_{2 p+2}\right)}{2 \eta}\right)\right]^{r}\right]^{1 / r} \\
& \leq\left[a_{3}\left(\sigma\left(x_{2 p+1}, x_{2 p+2}\right)\right)^{r}\right. \\
& \left.+a_{4}\left(\eta\left(\frac{\sigma\left(x_{2 p}, x_{2 p+1}\right)+\sigma\left(x_{2 p+1}, x_{2 p+2}\right)}{2 \eta}\right)\right)^{r}\right]^{1 / r} \\
& \leq\left[a_{3}\left(\sigma\left(x_{2 p+1}, x_{2 p+2}\right)\right)^{r}+a_{4}\left(\sigma\left(x_{2 p+1}, x_{2 p+2}\right)\right)^{r}\right]^{1 / r} \\
& =\left(a_{3}+a_{4}\right)^{1 / r} \sigma\left(x_{2 p+1}, x_{2 p+2}\right) \\
& =\sigma\left(x_{2 p+1}, x_{2 p+2}\right) \text { as } r \longrightarrow \infty .
\end{aligned}
$$

Therefore, using Lemma 9, we have

$$
\begin{aligned}
\sigma\left(x_{2 p+1}, x_{2 p+2}\right) & \leq H_{b}\left(S x_{2 p}, T x_{2 p+1}\right) \leq \varphi\left(\sigma\left(x_{2 p+1}, x_{2 p+2}\right)\right) \\
& <\sigma\left(x_{2 p+1}, x_{2 p+2}\right)
\end{aligned}
$$

a contradiction. It follows that for all $p \in \mathbb{N}$,

$$
\begin{aligned}
& x_{2 p}=x_{2 p+1} \in S x_{2 p}, \\
& x_{2 p}=x_{2 p+1}=x_{2 p+2} \in T x_{2 p+1}=T x_{2 p} .
\end{aligned}
$$

So, $x_{2 p}$ turns out to be the common fixed point of $S$ and $T$.

Again, for $r=0$ and $x_{2 p}=x_{2 p+1}$, for some $p \in \mathbb{N}$, we get $\mathscr{C}_{(S, T)}^{r}\left(x_{2 p}, x_{2 p+1}\right)=0$, for all $p \in \mathbb{N}$. Therefore, by property (ii) of $\Omega$, one obtains $\sigma\left(x_{2 p+1}, x_{2 p+2}\right)=0$, for all $p \in \mathbb{N}$, from which, on similar arguments as above, the same conclusion follows that $x_{2 p} \in S x_{2 p} \cap T x_{2 p}$. Hereafter, we presume that for all $p \in \mathbb{N}, x_{p+1} \neq x_{p}$ if and only if $\sigma\left(x_{p+1}, x_{p}\right)>0$.

Now, in view of (9), setting $x=x_{2 p}$ and $y=x_{2 p-1}$, we have

$$
\mathscr{C}_{(S, T)}^{r}\left(x_{2 p}, x_{2 p-1}\right)=\left\{\begin{array}{l}
{\left[a_{1}\left(\sigma\left(x_{2 p}, x_{2 p-1}\right)\right)^{r}+a_{2}\left(\sigma\left(x_{2 p}, S x_{2 p}\right)\right)^{r}+a_{3}\left(\sigma\left(x_{2 p-1}, T x_{2 p-1}\right)^{r}+a_{4}\left(\frac{\sigma\left(x_{2 p-1}, S x_{2 p}\right)+\sigma\left(x_{2 p}, T x_{2 p-1}\right)}{2 \eta}\right)^{r}\right]^{1 / r}, \quad \text { for } r>0,\right.} \\
\left(\sigma\left(x_{2 p}, x_{2 p-1}\right)\right)^{a_{1}}\left(\sigma\left(x_{2 p}, S x_{2 p}\right)\right)^{a_{2}}\left(\sigma\left(x_{2 p-1}, T x_{2 p-1}\right)\right)^{a_{3}} \times\left(\frac{\sigma\left(x_{2 p}, T x_{2 p-1}\right)+\sigma\left(x_{2 p-1}, S x_{2 p}\right)}{2 \eta}\right)^{a_{4}}, \quad \text { for } r=0 .
\end{array}\right.
$$


That is,

$\mathscr{C}_{(S, T)}^{r}\left(x_{2 p}, x_{2 p-1}\right)= \begin{cases}{\left[a_{1}\left(\sigma\left(x_{2 p}, x_{2 p-1}\right)\right)^{r}+a_{2}\left(\sigma\left(x_{2 p}, x_{2 p+1}\right)\right)^{r}+a_{3}\left(\sigma\left(x_{2 p-1}, x_{2 p}\right)\right)^{r}+a_{4}\left(\frac{\sigma\left(x_{2 p-1}, x_{2 p+1}\right)+\sigma\left(x_{2 p}, x_{2 p}\right)}{2 \eta}\right)^{r}\right]^{1 / r},} & \text { for } r>0, \\ \left(\sigma\left(x_{2 p}, x_{2 p-1}\right)\right)^{a_{1}}\left(\sigma\left(x_{2 p}, x_{2 p+1}\right)\right)^{a_{2}}\left(\sigma\left(x_{2 p-1}, x_{2 p}\right)\right)^{a_{3}} \times\left(\frac{\sigma\left(x_{2 p}, x_{2 p}\right)+\sigma\left(x_{2 p-1}, x_{2 p+1}\right)}{2 \eta}\right)^{a_{4}}, & \text { for } r=0\end{cases}$

Now, we consider the following two cases.

Case 1. $r>0$. Suppose that $\sigma\left(x_{2 p}, x_{2 p+1}\right) \geq \sigma\left(x_{2 p-1}, x_{2 p}\right)$, then from (16), we have

$$
\begin{aligned}
& \mathscr{C}_{(S, T)}^{r}\left(x_{2 p}, x_{2 p-1}\right) \\
& \leq {\left[a_{1}\left(\sigma\left(x_{2 p+1}, x_{2 p}\right)\right)^{r}+a_{2}\left(\sigma\left(x_{2 p+1}, x_{2 p}\right)\right)^{r}\right.} \\
&+a_{3}\left(\sigma\left(x_{2 p+1}, x_{2 p}\right)\right)^{r} \\
&\left.+a_{4}\left(\eta\left(\frac{\sigma\left(x_{2 p+1}, x_{2 p}\right)+\sigma\left(x_{2 p}, x_{2 p-1}\right)}{2 \eta}\right)\right)^{r}\right]^{1 / r} \\
& \leq {\left[a_{1}\left(\sigma\left(x_{2 p+1}, x_{2 p}\right)\right)^{r}+a_{2}\left(\sigma\left(x_{2 p+1}, x_{2 p}\right)\right)^{r}\right.} \\
&+a_{3}\left(\sigma\left(x_{2 p+1}, x_{2 p}\right)\right)^{r} \\
&\left.+a_{4}\left(\eta\left(\frac{\sigma\left(x_{2 p+1}, x_{2 p}\right)+\sigma\left(x_{2 p+1}, x_{2 p}\right)}{2 \eta}\right)\right)^{r}\right]^{1 / r} \\
& \leq {\left[a_{1}\left(\sigma\left(x_{2 p+1}, x_{2 p}\right)\right)^{r}+a_{2}\left(\sigma\left(x_{2 p+1}, x_{2 p}\right)\right)^{r}\right.} \\
&\left.+a_{3}\left(\sigma\left(x_{2 p+1}, x_{2 p}\right)\right)^{r}+a_{4}\left(\sigma\left(x_{2 p+1}, x_{2 p}\right)\right)^{r}\right]^{1 / r} \\
&= {\left[\left(a_{1}+a_{2}+a_{3}+a_{4}\right) \sigma\left(x_{2 p+1}, x_{2 p}\right)^{r}\right]^{1 / r} } \\
&= \sigma\left(x_{2 p+1}, x_{2 p}\right)\left(\sum_{i=1}^{4} a_{i}\right)^{1 / r}=\sigma\left(x_{2 p+1}, x_{2 p}\right) .
\end{aligned}
$$

Hence, from (7) and (17), we have

$$
\sigma\left(x_{2 p+1}, x_{2 p}\right) \leq \varphi\left(\sigma\left(x_{2 p+1}, x_{2 p}\right)\right) .
$$

Since $\varphi$ is a $b$-comparison function, therefore, (18) implies that

$$
\sigma\left(x_{2 p+1}, x_{2 p}\right)<\sigma\left(x_{2 p+1}, x_{2 p}\right) \text {, }
$$

which is a contradiction. Consequently, it follows that $\sigma\left(x_{2 p+1}, x_{2 p}\right) \leq \sigma\left(x_{2 p}, x_{2 p-1}\right)$. Thus, from (18), we obtain

$$
\sigma\left(x_{2 p+1}, x_{2 p}\right) \leq \varphi\left(\sigma\left(x_{2 p}, x_{2 p-1}\right)\right) .
$$

Setting $n=2 p \in \mathbb{N}$ in (20) yields

$$
\begin{aligned}
\sigma\left(x_{n+1}, x_{n}\right) & \leq \varphi\left(\sigma\left(x_{n}, x_{n-1}\right)\right) \leq \varphi^{2}\left(\sigma\left(x_{n-1}, x_{n-2}\right)\right) \\
& \leq \varphi^{3}\left(\sigma\left(x_{n-2}, x_{n-3}\right)\right) \vdots \quad \vdots \quad \leq \varphi^{n}\left(\sigma\left(x_{1}, x_{0}\right)\right) .
\end{aligned}
$$

From (21), by triangle inequality on $(X, \sigma, \eta)$, for all $k \geq 1$, we have

$$
\begin{aligned}
\sigma\left(x_{n+k}, x_{n}\right) & \leq \eta\left(\sigma\left(x_{n+k}, x_{n+1}\right)+\sigma\left(x_{n+1}, x_{n}\right)\right) \\
& \leq \frac{1}{\eta^{n-1}} \sum_{i=n}^{n+k-1} \eta^{k} \sigma\left(x_{i}, x_{i+1}\right) \\
& \leq \frac{1}{\eta^{n-1}} \sum_{i=n}^{n+k-1} \eta^{k} \varphi^{k}\left(\sigma\left(x_{1}, x_{0}\right)\right) \\
& \leq \frac{1}{\eta^{n-1}} \sum_{i=n}^{\infty} \eta^{i} \varphi^{i}\left(\sigma\left(x_{1}, x_{0}\right)\right) .
\end{aligned}
$$

Letting $n \longrightarrow \infty$ in (22) and applying Lemma 10, we find that $\lim _{n \rightarrow \infty} \sigma\left(x_{n+k}, x_{n}\right)=0$. Therefore, $\left\{x_{n}\right\}_{n \in \mathbb{N}}$ is a Cauchy sequence of points of $(X, \sigma, \eta)$. The completeness of this space implies that there exists $u \in X$ such that

$$
\lim _{n \rightarrow \infty} \sigma\left(x_{n}, u\right)=0 .
$$

Now, we show that $u$ is the expected common fixed point of $S$ and $T$. First, assume that $u \notin S u$ so that $\sigma(u, S u)>0$. Then, by Lemma 12 and the case $r>0$ in the contractive inequality (7), we have

$$
\begin{aligned}
\sigma(u, S u) \leq & \eta \sigma\left(u, x_{n}\right)+\eta \sigma\left(x_{n}, S u\right) \\
\leq & \left.\eta \sigma\left(u, x_{n}\right)+\eta H_{b}\left(S u, T x_{n-1}\right)\right) \\
\leq & \eta \sigma\left(u, x_{n}\right)+\eta \varphi\left(\mathscr{C}_{(S, T)}^{r}\left(u, x_{n-1}\right)\right) \\
= & \eta \sigma\left(u, x_{n}\right)+\eta \varphi\left(\left[a_{1}\left(\sigma\left(u, x_{n-1}\right)\right)^{r}\right.\right. \\
& +a_{2}(\sigma(u, S u))^{r}+a_{3}\left(\sigma\left(x_{n-1}, T x_{n-1}\right)\right)^{r} \\
& \left.\left.+a_{4}\left(\frac{\sigma\left(x_{n-1}, S u\right)+\sigma\left(u, T x_{n-1}\right)}{2 \eta}\right)^{r}\right]^{1 / r}\right)
\end{aligned}
$$




$$
\begin{gathered}
=\eta \sigma\left(u, x_{n}\right)+\eta \varphi\left(\left[a_{1}\left(\sigma\left(u, x_{n-1}\right)\right)^{r}\right.\right. \\
+a_{2}(\sigma(u, S u))^{r}+a_{3}\left(\sigma\left(x_{n-1}, x_{n}\right)\right)^{r} \\
\left.\left.+a_{4}\left(\frac{\sigma\left(x_{n-1}, S u\right)+\sigma\left(u, x_{n}\right)}{2 \eta}\right)^{r}\right]^{1 / r}\right) .
\end{gathered}
$$
give

Letting $n \longrightarrow \infty$ in (24) and using the properties of $\varphi \in \Omega$

$$
\sigma(u, S u)<\eta \sigma(u, S u)\left(a_{2}+a_{4}\right)^{1 / r}
$$

and as $r \longrightarrow \infty$,

$$
\sigma(u, S u)<\eta \sigma(u, S u)
$$

Notice that taking $\eta=1$ in (26) yields a contradiction. Thus, $\sigma(u, S u)=0$, which further implies that $u \in S u$. On similar steps, by assuming that $u$ is not a fixed point of $T$ and considering

$$
\begin{aligned}
\sigma(u, T u) & \leq \eta \sigma\left(u, x_{n}\right)+\eta \sigma\left(x_{n}, T u\right) \\
& \leq \eta \sigma\left(\mathrm{u}, x_{n}\right)+\eta H_{b}\left(S x_{n-1}, T u\right) \\
& \leq \eta \sigma\left(u, x_{n}\right)+\eta \varphi\left(\mathscr{C}_{(S, T)}^{r}\left(x_{n-1}, u\right)\right),
\end{aligned}
$$

we can show that $u \in T u$. Consequently, for $r>0$, there exists $u \in X$ such that $u \in S u \cap T u$.

Case 2. $r=0$. Using the inequality (16) on account of $b$-comparison of $\varphi$, we have

$$
\begin{aligned}
& \sigma\left(x_{2 p}, x_{2 p-1}\right) \leq H_{b}\left(S x_{2 p-1}, T x_{2 p-2}\right) \leq \varphi\left(\mathscr{C}_{(S, T)}^{r}\left(x_{2 p-1}, x_{2 p-2}\right)\right) \\
&<\left(\sigma\left(x_{2 p-1}, x_{2 p-2}\right)\right)^{a_{1}}\left(x_{2 p-1}, S x_{2 p-1}\right) a_{2}^{a_{2}}\left(\sigma\left(x_{2 p-2}, T x_{2 p-2}\right)\right)^{a_{3}} \\
& \times\left(\frac{\sigma\left(x_{2 p-1}, T x_{2 p-2}\right)+\sigma\left(x_{2 p-2}, S x_{2 p-1}\right)}{2 \eta}\right)^{a_{4}} \\
&=\left(\sigma\left(x_{2 p-1}, x_{2 p-2}\right)\right)^{a_{1}}\left(\sigma\left(x_{2 p-1}, x_{2 p}\right)\right)^{a_{2}}\left(\sigma\left(x_{2 p-2}, x_{2 p-1}\right)\right)^{a_{3}} \\
&\left.\left.\times\left(\frac{\sigma\left(x_{2 p-1}, x_{2 p-1}\right)+\sigma\left(x_{2 p-2}, x_{2 p}\right)}{2 \eta}\right)^{a_{4}}\right)\right)^{a_{2}}\left(\sigma\left(x_{2 p-2}, x_{2 p-1}\right)\right)^{a_{3}} \\
& \leq\left(\sigma\left(x_{2 p-1}, x_{2 p-2}\right)\right)^{a_{1}}\left(\sigma\left(x_{2 p-1}, x_{2 p}\right)\right. \\
& \times\left(\frac{\sigma\left(x_{2 p}, x_{2 p-1}\right)+\sigma\left(x_{2 p-1}, x_{2 p-2}\right)}{2}\right)^{a_{4}} \\
&=\left(\sigma\left(x_{2 p-1}, x_{2 p-2}\right)\right)^{a_{1}+a_{3}}\left(\sigma\left(x_{2 p-1}, x_{2 p}\right)\right)^{a_{2}} \\
& \times\left(\frac{\sigma\left(x_{2 p}, x_{2 p-1}\right)+\sigma\left(x_{2 p-1}, x_{2 p-2}\right)}{2}\right)^{1-a_{1}-a_{2}-a_{3}} .
\end{aligned}
$$

Assume that $\sigma\left(x_{2 p-1}, x_{2 p-2}\right) \leq \sigma\left(x_{2 p}, x_{2 p-1}\right)$; then, (28) gives

$$
\begin{aligned}
\sigma\left(x_{2 p}, x_{2 p-1}\right) & \leq \varphi\left(\mathscr{C}_{(S, T)}^{r}\left(x_{2 p-1}, x_{2 p-2}\right)\right) \\
& <\left(\sigma\left(x_{2 p}, x_{2 p-1}\right)\right)^{a_{1}+a_{2}+a_{3}}\left(\sigma\left(x_{2 p}, x_{2 p-1}\right)\right)^{1-a_{1}-a_{2}-a_{3}} \\
& =\sigma\left(x_{2 p}, x_{2 p-1}\right),
\end{aligned}
$$

a contradiction. Therefore,

$$
\sigma\left(x_{2 p}, x_{2 p-1}\right) \leq \sigma\left(x_{2 p-1}, x_{2 p-2}\right) .
$$

Using (28) and (30), we obtain

$$
\sigma\left(x_{2 p}, x_{2 p-1}\right) \leq \varphi\left(\sigma\left(x_{2 p-1}, x_{2 p-2}\right)\right)
$$

Notice that (31) is equivalent to (21). So, on similar steps, we deduce that the sequence $\left\{x_{n}\right\}_{n \in \mathbb{N}}$ is Cauchy in $(X, \sigma, \eta)$. Thus, the completeness of this space guarantees that $\sigma\left(x_{n}, u\right) \longrightarrow 0$ as $n \longrightarrow \infty$, for some $u \in X$.

To see that $u$ is a common fixed point of $T$ and $S$, we apply Lemma 12 and inequality (7) as follows:

$$
\begin{aligned}
& \sigma(u, T u) \leq \eta \sigma\left(u, x_{n}\right)+\eta \sigma\left(x_{n}, T u\right) \leq \eta \sigma\left(u, x_{n}\right) \\
& +\eta H_{b}\left(S x_{n-1}, T u\right) \leq \eta \sigma\left(u, x_{n}\right)+\eta \varphi\left(\mathscr{C}_{(S, T)}^{r}\left(x_{n-1}, u\right)\right),
\end{aligned}
$$

where

$\mathscr{C}_{(S, T)}^{r}\left(x_{n-1}, u\right)=\left(\sigma\left(x_{n-1}, u\right)\right)^{a_{1}}\left(\sigma\left(x_{n-1}, S x_{n-1}\right)\right)^{a_{2}} \quad(\sigma(u$, $T u))^{a_{3}} \times\left(\sigma\left(x_{n-1}, T u\right)+\sigma\left(u, S x_{n-1}\right) / 2 \eta\right)^{a_{4}}=\left(\sigma\left(x_{n-1}, u\right)\right)^{a_{1}}(\sigma$ $\left.\left(x_{n-1}, x_{n}\right)\right)^{a_{2}}(\sigma(u, T u))^{a_{3}} \times\left(\sigma\left(x_{n-1}, T u\right)+\sigma\left(u, x_{n}\right) / 2 \eta\right)^{a_{4}}$. $(33)(70)$

We see that $\lim _{n \rightarrow \infty} \mathscr{C}_{(S, T)}^{r}\left(x_{n-1}, u\right)=0$. Hence, under this limiting case, (33) becomes

$$
\sigma(u, T u) \leq \eta \varphi(0)
$$

By condition (ii) of $\varphi$, (33) implies that $\sigma(u, T u)=0$. Therefore, $u \in T u$. On similar steps, we can show that $u \in S$ $u$. Consequently, $S$ and $T$ have a common fixed point $u \in X$.

Corollary 15. Let $(X, \sigma, \eta)$ be a complete b-metric space and $S: X \longrightarrow \mathscr{P}^{r}(X)$ be a multivalued map. Suppose that for each $x \in X, S x$ is a nonempty bounded proximal subset of $X$. If for all $x, y \in X$, the following conditions are satisfied:

$$
H_{b}(S x, S y) \leq \varphi\left(\mathscr{C}_{(S)}^{r}(x, y)\right)
$$

where $\varphi \in \Omega, r \geq 0$, and $a_{i} \geq 0, i=1,2,3,4$ with $\sum_{i=1}^{4} a_{i}=1$ and 


$$
\mathscr{C}_{(S)}^{r}(x, y)=\left\{\begin{array}{l}
{\left[a_{1}(\sigma(x, y))^{r}+a_{2}\left(\sigma(x, S x)^{r}+a_{3}\left(\sigma(y, S y)^{r}+a_{4}\left(\frac{\sigma(y, S x)+\sigma(x, S y)}{2 \eta}\right)^{r}\right]^{1 / r}, \quad \text { for } r>0, x, y \in X,\right.\right.} \\
\sigma(x, y))^{a_{1}}(\sigma(x, S x))^{a_{2}}(\sigma(y, S y))^{a_{3}}\left(\frac{\sigma(x, S y)+\sigma(y, S x)}{2 \eta}\right)^{a_{4}}, \quad \text { for } r=0, x, y \in X \backslash \mathscr{F}_{i x}(S),
\end{array}\right.
$$

where

$$
\mathscr{F}_{i x}(S)=\{x \in X: x \in S x\}
$$

then, there exists $u \in X$ such that $u \in S u$.

\section{Proof. Put $S=T$ in Theorem 14.}

The following example is provided to support the hypotheses of Theorem 14 for $S=T$.

Example 3. Let $X=\{1,2,3\}$ and $\sigma(x, y)=|x-y|^{2}$, for all $x$, $y \in X$. Then, $(X, \sigma, \eta=2)$ is a complete $b$-metric space. Note that $(X, \sigma, \eta=2)$ is not a metric space, since for $x=1, y=3$, and $z=2$, we have

$$
\sigma(x, y)=4>2=\sigma(x, z)+\sigma(z, y)
$$

Define a multivalued map $S: X \longrightarrow \mathscr{P}^{r}(X)$ by

$$
S x= \begin{cases}\{1,2\}, & \text { if } x=1, \\ \{2,3\}, & \text { if } x=2, \\ \{2\}, & \text { if } x=3 .\end{cases}
$$

Let $\varphi(t)=t / 2$, for all $t \geq 0$. Clearly, $\varphi \in \Omega$. Now, we verify inequality (7) as follows: For $r>0$ and $x, y \in X$ with $x \neq y$, consider the following cases.

Case 1. $x=1$ and $y=2$. For this, we have $H_{b}(S 1, S 2)=H_{b}$ $(\{1,2\},\{2,3\})=1 ; \sigma(1,2)=\sigma(1, S 2)=1 ;$ and $\sigma(1, S 1)=\sigma$ $(2, S 2)=\sigma(2, S 1)=0$. Define $\Delta_{\sigma_{1}}(1,2)$ as

$$
\begin{aligned}
\Delta_{\sigma_{1}}(1,2)= & {\left[a_{1}(\sigma(1,2))^{r}+a_{2}(\sigma(1, S 1))^{r}+a_{3}(\sigma(2, S 2))^{r}\right.} \\
& \left.+a_{4}\left(\frac{\sigma(2, S 1)+\sigma(1, S 2)}{2 \times 2}\right)^{r}\right]^{1 / r}
\end{aligned}
$$

Take $a_{1}=a_{4}=1 / 2$ and $a_{2}=a_{3}=0$; then, (39) becomes

$$
\begin{aligned}
\Delta_{\sigma_{1}}(1,2)= & {\left[\frac{1}{2}+\frac{1}{2}\left(\frac{1}{2 \times 2}\right)^{r}\right]^{1 / r}=\left(\frac{1}{2}\right)^{1 / r}+\frac{1}{r}\left(\frac{1}{2}\right)^{(1 / r)-1} } \\
& \times \frac{1}{2}\left(\frac{1}{2 \times 2}\right)^{r}+\cdots+\left(\frac{1}{2}\right)^{1 / r}\left(\frac{1}{2 \times 2}\right) \\
= & \frac{1}{2}\left(\frac{1}{2}\right)^{(1 / r)-1}+\frac{1}{2 r}\left(\frac{1}{2}\right)^{(1 / r)-1}\left(\frac{1}{2 \times 2}\right)^{r} \\
& +\cdots+\frac{1}{2}\left(\frac{1}{2}\right)^{(1 / r)-1}\left(\frac{1}{2 \times 2}\right)
\end{aligned}
$$

Letting $r \longrightarrow \infty^{+}$in $(40)$, we have

$$
\Delta_{\sigma_{1}}(1,2)=\frac{1}{2}\left(2+\frac{1}{2}\right)=\varphi\left(C_{(S)}^{r}(1,2)\right) .
$$

Therefore,

$$
H_{b}(T 1, T 2)=1 \leq \varphi\left(C_{(S)}^{r}(1,2)\right)
$$

Case 2. For $x=1$ and $y=3$, we have

$$
H_{b}(T 1, T 3)=H_{b}(\{1,2\},\{2\})=1,
$$

and $\sigma(1,3)=4 ; \sigma(1, S 1)=0$; and $\sigma(3, S 3)=\sigma(3, S 1)=\sigma(1$, $S 3)=1$. Define $\Delta_{\sigma_{2}}(1,3)$ as

$$
\begin{aligned}
\Delta_{\sigma_{2}}(1,3)= & {\left[a_{1}(\sigma(1,3))^{r}+a_{2}(\sigma(1, S 1))^{r}+a_{3}(\sigma(2, S 3))^{r}\right.} \\
& \left.+a_{4}\left(\frac{\sigma(3, S 1)+\sigma(1, S 3)}{2 \times 2}\right)^{r}\right]^{1 / r} .
\end{aligned}
$$

Take $a_{1}=a_{4}=1 / 2$ and $a_{2}=a_{3}=0$; then,

$$
\begin{aligned}
\Delta_{\sigma_{2}}(1,3) & =\left[\frac{1}{2}(4)^{r}+\frac{1}{2}\left(\frac{1+1}{2 \times 2}\right)^{r}\right]^{1 / r} \\
& =\frac{1}{2}\left(\frac{1}{2}\right)^{(1 / r)-1}\left[4^{r}+\left(\frac{1}{2}\right)^{r}\right]^{1 / r} \\
& =\frac{1}{2}\left(\frac{1}{2}\right)^{(1 / r)-1}\left[4+\cdots+\frac{1}{2}\right]=\varphi\left(C_{(S)}^{r}(1,3)\right) .
\end{aligned}
$$


Thus, $H_{b}(S 1, S 3)=1 \leq \varphi\left(C_{(S)}^{r}(1,3)\right)$. Consequently, for all $r>0$ and $x, y \in X$ with $x \neq y$, we have

$$
H_{b}(S x, S y)=1 \leq \varphi\left(C_{(S)}^{r}(x, y)\right)
$$

Now, we check the case for $r=0$ and $x, y \in X \backslash \mathscr{F}_{i x}(S)$. Obviously, $x, y \in\{3\}$, and

$$
H_{b}(S x, S y)=H_{b}(S 3, S 3)=H_{b}(\{2\},\{2\})=0 \leq \varphi\left(C_{(S)}^{r}(x, y)\right) .
$$

Hence, all the hypotheses of Theorem 14 are satisfied with $S=T$. We can see that the set of all fixed points of $S$ is given by $\mathscr{F}_{i x}(S)=\{1,2\}$.

Corollary 16. Let $(X, \sigma, \eta)$ be a complete b-metric space and $S: X \longrightarrow \mathscr{P}^{r}(X)$ be a multivalued map. Suppose that for each $x \in X, S x$ is a nonempty bounded proximal subsets of $X$. If

$$
H_{b}(S x, S y) \leq \varphi\left(\frac{1}{4} \mathscr{C}_{(S)}^{r}(x, y)\right)
$$

for all $x, y \in X$, where $\varphi \in \Omega$ and

$$
\mathscr{C}_{(S)}^{r}=\sigma(x, y)+\sigma(x, S x)+\sigma(y, S y)+\frac{\sigma(y, S x)+\sigma(x, S y)}{2 \eta},
$$

then, there exists $u \in X$ such that $u \in S u$.

Proof. Take $S=T, r=1$, and $a_{1}=a_{2}=a_{3}=a_{4}=1 / 4$ in Theorem 14

Corollary 17. Let $(X, \sigma, \eta)$ be a complete b-metric space and $S, T: X \longrightarrow \mathscr{P}^{r}(X)$ be multivalued maps. Suppose that for each $x, y \in X, S x$ and Ty are nonempty bounded proximal subsets of $X$. If there exists $\lambda \in[0,1)$ such that

$$
\begin{aligned}
& H_{b}(S x, T y) \\
& \quad \leq \lambda\left(\sqrt[4]{(\sigma(x, y)) \sigma(x, S x)(\sigma(y, T y))\left(\frac{\sigma(x, T y)+\sigma(y, S x)}{2 \eta}\right)}\right),
\end{aligned}
$$

then $S$ and $T$ have a common fixed point in $X$.

Proof. Take $a_{1}=a_{2}=a_{3}=a_{4}=1 / 4$ and $\varphi(t)=\lambda t$ for all $t \geq 0$ and $r=0$ in Theorem 14 .

Corollary 18. Let $(X, \sigma, \eta)$ be a complete b-metric space and $S: X \longrightarrow \mathscr{P}^{r}(X)$ be a multivalued map. Suppose that for each $x \in X, S x$ is a nonempty bounded proximal subset of $X$. If there exists $\lambda \in[0,1)$ such that

$$
H_{b}(S x, S y) \leq \lambda \sigma(x, y)
$$

then, there exists $u \in X$ such that $u \in S u$.

Proof. Put $S=T, a_{1}=r=1, a_{2}=a_{3}=a_{4}=0$, and $\varphi(t)=\lambda t$, $t \geq 0$, in Theorem 14 .

In the following corollary, as an application of Corollary 15, we deduce the main result of Karapinar and Fulga [17] without using the continuity of the considered singlevalued mapping.

Corollary 19. (see [17], Theorem 1). Let $(X, \sigma, \eta)$ be a complete $b$-metric space and $F: X \longrightarrow X$ be a single-valued mapping. If

$$
\sigma(F x, F y) \leq \varphi\left(\mathscr{C}_{F}^{r}(x, y)\right)
$$

for all $x, y \in X$, where $\varphi \in \Omega$ and $r \geq 0, a_{i} \geq 0, i=1,2,3,4$, with $\sum_{i=1}^{4} a_{i}=1$ and

$$
\mathscr{C}_{F}^{r}(x, y)=\left\{\begin{array}{l}
{\left[a_{1}(\sigma(x, y))^{r}+a_{2}(\sigma(x, F x))^{r}+a_{3}(\sigma(y, F y))^{r}+a_{4}\left(\frac{\sigma(y, F x)+\sigma(x, F y)}{2 \eta}\right)^{r}\right]^{1 / r}, \quad \text { for } r>0, x, y \in X,} \\
(\sigma(x, y))^{a_{1}}(\sigma(x, F x))^{a_{2}}(\sigma(y, F y))^{a_{3}}\left(\frac{\sigma(x, F y)+\sigma(y, F x)}{2 \eta}\right)^{a_{4}}, \quad \text { for } r=0, x, y \in X \backslash \mathscr{F}_{i x}(F),
\end{array}\right.
$$

where

$$
\mathscr{F}_{i x}(F)=\{x \in X: x=F x\} .
$$

Then, there exists $u \in X$ such that $u=F u$.
Proof. We know that $\{x\} \in \mathscr{K}(X) \subseteq \mathscr{P}_{b}^{r}(X)$ for every $x \in X$. Consider a mapping $\Xi: X \longrightarrow \mathscr{P}^{r}(X)$ defined as $\Xi x=\{F x\}$, $x \in X$. Then, all the conditions of Corollary 15 reduce to the conditions of Corollary 19 with $S=F$ and $H_{b}(S x, S y)=$ $\sigma(F x, F y)$, for all $x, y \in X$. Thus, by application of Corollary 15 , there exists $u \in X$ such that $\{u\}=\Xi u$. The definition of $\Xi$ implies that $\Xi u=\{F u\}$. Consequently, $u=F u$. 
Proof. Alternative proof of Corollary 19.

Let $F: X \longrightarrow X$ be the single-valued mapping in Corollary 19; then, define a multivalued mapping $S: X \longrightarrow$ $\mathscr{P}^{r}(X)$ by $S x=\{F x\}$, for all $x \in X$. Clearly, $S x \in \mathscr{P}_{b}^{r}(X)$. Consequently, Corollary 15 can be applied to find $u \in X$ such that $u \in S u=\{F u\}$, which further implies that $F u=u$.

Remark 20. It is clear that if we take $\eta=1$ in all the above results, we can deduce their analogs in the setting of metric spaces.

\section{Application to Fredholm Integral Inclusions}

In this section, we apply one of the results in the previous section to study some sufficient conditions for existence of solutions of a Fredholm Integral inclusion. For basic concepts of integral inclusions, we refer the interested reader to $[22,23,25]$ and references therein. type:

Consider the following integral inclusion of Fredholm

$$
x(t) \in\left[f(t)+\int_{a}^{b} L(t, s, x(s)) \sigma s,\right]
$$

for $t \in[a, b]$, where $x \in C([a, b], \mathbb{R})$ is an unknown function, $f \in C([a, b], \mathbb{R})$ is a given real-valued function, and $L:[a, b]$ $\times[a, b] \times \mathbb{R} \longrightarrow F_{c v}(\mathbb{R})$ is a given multivalued map, where we denote the family of nonempty compact and convex subsets of $\mathbb{R}$ by $F_{c v}(\mathbb{R})^{1}$. The set of all real-valued continuous functions on $[a, b]$ shall be represented by $C([a, b], \mathbb{R})$.

Now, we study the existence of solutions of (55) under the following assumptions.

\section{Theorem 21. Suppose that}

$\left(C_{1}\right)$ : the multivalued map $L:[a, b] \times[a, b] \times \mathbb{R} \longrightarrow F_{c v}$ $(\mathbb{R})$ is such that for every $x \in C([a, b], \mathbb{R})$, the map $L_{x}(t, s)$ $:=L(t, s, x(s))$ is lower semicontinuous.

$\left(C_{2}\right)$ : there exists a b-comparison function $\varphi: \mathbb{R}_{+} \longrightarrow \mathbb{R}_{+}$ such that for all $x, y \in C([a, b], \mathbb{R})$,

$$
\begin{aligned}
H_{b}\left(L_{x}(t, s), L_{y}(t, s)\right) \\
\leq \quad \pi(t, s) \varphi\left(\frac { 1 } { 4 } \left(|x(s)-y(s)|^{2}\right.\right. \\
\quad+|x-L(t, s, x(s))|^{2}+|y(s)-L(t, s, y(s))|^{2} \\
\left.\left.\quad+\frac{|y(s)-L(t, s, x(s))|^{2}+|x(s)-L(t, s, y(s))|^{2}}{2 \eta}\right)\right),
\end{aligned}
$$

for each $t, s \in[a, b]$ and $\sup \left(\int_{a}^{b} \pi(t, s)\right)^{2} \sigma s \leq 1$, where $\pi(t,$. $\in L^{1}[a, b]$ and for some constants $\eta \geq 1$.

Then, the integral inclusion (55) has at least one solution in $C([a, b], \mathbb{R})$.
Proof. Let $X=C([a, b], \mathbb{R})$ and $\sigma: X \times X \longrightarrow \mathbb{R}_{+}$be defined by

$$
\sigma(x, y)=|x-y|^{2}, \quad \text { for all } x, y \in X
$$

Then, $(X, \sigma, \eta)$ is a complete $b$-metric space with the parameter $\eta=2$. Note that $X$ endowed with this metric $\sigma$ is not a metric space. Let $S: X \longrightarrow \mathscr{P}^{r}(X)$ be a multivalued map defined as

$S x=\left\{y \in X: y(t) \in f(t)+\int_{a}^{b} L(t, s, x(s)) \sigma s, t \in[a, b]\right\}$.

Obviously, the set of solutions of (55) coincides with the set of fixed points of $S$. Therefore, we have to show that under the given suppositions, $S$ has at least one fixed point in $X$. For this, we shall verify that all the hypotheses of Corollary 16 are satisfied.

Let $x \in X$ be arbitrary. Since the multivalued map $L_{x}$ : $[a, b] \times[a, b] \longrightarrow \sqsubseteq_{c v}(\mathbb{R})$ is lower semicontinuous, it follows from Michael's selection theorem ([33], Theorem 1]) that there exists a continuous map $\rho_{x}:[a, b] \times[a, b] \longrightarrow \mathbb{R}$ such that $\rho_{x}(t, s) \in L_{x}(t, s)$, for each $(t, s) \in[a, b] \times[a, b]$. Therefore, $f(t)+\int_{a}^{b} \rho_{x}(t, s) \sigma s \in S x$. So, $S x$ is nonempty. One can easily see that $S x$ is a closed subset of $X$. Further, since $f \in C([a, b], \mathbb{R})$ and $L_{x}(t, s)$ is continuous on $[a, b] \times[a, b]$, their range sets are compact. Hence, $S x \in \mathscr{K}(X) \subseteq \mathscr{P}_{b}^{r}(X)$ $\subseteq \mathscr{P}^{r}(X)$.

Take $x_{1}, x_{2} \in X$; then $S x_{1}$ and $S x_{2}$ are nonempty bounded proximal subsets of $X$. Let $y_{1} \in S x_{1}$ be arbitrary such that

$$
y_{1}(t) \in f(t)+\int_{a}^{b} L\left(t, s, x_{1}(s)\right) \sigma s, \quad t \in[a, b] .
$$

This means for each $(t, s) \in[a, b] \times[a, b]$, there exists $\rho_{x_{1}}$ $\in L_{x_{1}}(t, s)$ such that

$$
y_{1}(t)=f(t)+\int_{a}^{b} \rho_{x_{1}}(t, s) \sigma s, t \in[a, b] .
$$

Since from $\left(\mathrm{C}_{2}\right)$,

$$
\begin{aligned}
& H_{b}\left(L\left(t, s, x_{1}(s)\right), L\left(t, s, x_{2}(s)\right)\right) \\
& \leq \pi(t, s) \varphi\left(\frac { 1 } { 4 } \left(\left|x_{1}(s)-x_{2}(s)\right|^{2}\right.\right. \\
& \quad+\left|x_{1}(s)-L\left(t, s, x_{1}(s)\right)\right|^{2}+\left|x_{2}(s)-L\left(t, s, x_{2}(s)\right)\right|^{2} \\
&\left.\left.\quad+\frac{\left|x_{2}(s)-L\left(t, s, x_{1}(s)\right)\right|^{2}+\left|x_{1}-L\left(t, s, x_{2}(s)\right)\right|^{2}}{2 \eta}\right)\right),
\end{aligned}
$$

for each $t, s \in[a, b]$ and all constants $\eta \geq 1$, so, there exists $\rho_{x_{2}} \in L_{x_{2}}(t, s)$ such that 


$$
\begin{aligned}
\left|\rho_{x_{1}}(t, s)-\rho_{x_{2}}(t, s)\right|^{2} & \\
\leq & \pi(t, s) \varphi\left(\frac { 1 } { 4 } \left(\left|x_{1}(s)-x_{2}(s)\right|^{2}\right.\right. \\
& +\left|x_{1}(s)-L\left(t, s, x_{1}(s)\right)\right|^{2}+\left|x_{2}(s)-L\left(t, s, x_{2}(s)\right)\right|^{2} \\
& \left.\left.+\frac{\left|x_{2}(s)-L\left(t, s, x_{1}(s)\right)\right|^{2}+\left|x_{1}-L\left(t, s, x_{2}(s)\right)\right|^{2}}{2 \eta}\right)\right),
\end{aligned}
$$

for all $(t, s) \in[a, b] \times[a, b]$.

Now, consider the multivalued map $\mathfrak{M}$ defined by

$$
\begin{aligned}
\mathfrak{M}(t, s) & =L_{x_{2}}(t, s) \cap\left\{\omega \in \mathbb{R}:\left|\rho_{x_{1}}(t, s)-\omega\right|^{2}\right. \\
& \left.\leq \pi(t, s) \varphi\left(\frac{1}{4}\left|x_{1}(s)-x_{2}(s)\right|^{2}\right)\right\} .
\end{aligned}
$$

Taking into account the fact that from $\left(\mathrm{C}_{1}\right), \mathfrak{M}$ is lower semicontinuous, therefore, there exists a continuous map $\rho_{x_{2}}:[a, b] \times[a, b] \longrightarrow \mathbb{R}$ such that $\rho_{x_{2}}(t, s) \in \mathfrak{M}(t, s)$, for all $(t, s) \in[a, b] \times[a, b]$. Then,

$y_{2}(t)=f(t)+\int_{a}^{b} \rho_{x_{1}}(t, s) \sigma s \in f(t)+\int_{a}^{b} L\left(t, s, x_{2}(s)\right) \sigma s, \quad t \in[a, b]$.

Thus, $y_{2} \in S x_{2}$, and

$$
\begin{aligned}
\mid y_{1} & (t)-\left.y_{2}(t)\right|^{2} \leq\left(\int_{a}^{b}\left|\rho_{x_{1}}(t, s)-\rho_{x_{2}}(t, s)\right| \sigma s\right)^{2} \\
& \leq \sup \left(\int_{a}^{b} \pi(t, s) \sigma s\right) \varphi\left(\frac { 1 } { 4 } \left(\left|x_{1}(s)-x_{2}(s)\right|^{2}\right.\right. \\
& +\left|x_{1}(s)-L\left(t, s, x_{1}(s)\right)\right|^{2}+\left|x_{2}(s)-L\left(t, s, x_{2}(s)\right)\right|^{2} \\
& \left.\left.+\frac{\left.\left|x_{2}(s)-L\left(t, s, x_{1}(s)\right)\right|^{2}+\left|x_{1}(s)-L\left(t, s, x_{2}(s)\right)\right|^{2}\right)}{2 \eta}\right)\right) \\
& \leq \varphi\left(\frac { 1 } { 4 } \left(\left|x_{1}(s)-x_{2}(s)\right|^{2}+\left|x_{1}(s)-L\left(t, s, x_{1}(s)\right)\right|^{2}\right.\right. \\
& +\left|x_{2}(s)-L\left(t, s, x_{2}(s)\right)\right|^{2} \\
& \left.\left.+\frac{\left|x_{2}(s)-L\left(t, s, x_{1}(s)\right)\right|^{2}+\left|x_{1}(s)-L\left(t, s, x_{2}(s)\right)\right|^{2}}{2 \eta}\right)\right) \\
& \leq \varphi\left(\frac{1}{4}\left(\sigma\left(x_{1}, x_{2}\right)+\sigma\left(x_{1}, S x_{1}\right)+\sigma\left(x_{2}, S x_{2}\right)\right)\right. \\
& \left.\left.+\frac{\sigma\left(x_{2}, S x_{1}\right)+\sigma\left(x_{1}, S x_{2}\right)}{2 \eta}\right)\right) .
\end{aligned}
$$

Therefore, $\sigma\left(y_{1}, y_{2}\right) \leq \varphi\left((1 / 4) C_{(S)}^{r}\left(x_{1}, x_{2}\right)\right)$; that is,

$$
H_{b}\left(S x_{1}, S x_{2}\right) \leq \varphi\left(\frac{1}{4} C_{(S)}^{r}\left(x_{1}, x_{2}\right)\right)
$$

Setting $x=x_{1}$ and $y=x_{2}$ in the above inequation gives

$$
\begin{aligned}
C_{S}^{r}\left(x_{1}, x_{2}\right)= & C_{S}^{r}(x, y)=\sigma(x, y) \\
& +\sigma(x, S x)+\sigma(y, S y) \\
& +\frac{\sigma(y, S x)+\sigma(x, S y)}{2 \eta}
\end{aligned}
$$

and $H_{b}(S x, S y) \leq \varphi\left((1 / 4) C_{(S)}^{r}(x, y)\right)$. Thus, all the hypotheses of Corollary 16 are satisfied. It follows that the integral inclusion (55) has at least one solution in $X$.

Example 4. Let $L:[0,20] \times[0,20] \times \mathbb{R} \longrightarrow F_{c v}(\mathbb{R})$ be defined by

$$
L(t, s, x(s))=\left(\begin{array}{ll}
{\left[\frac{-1}{6}, 4\right],} & \text { if } x \neq 0,(s, t) \in[0,3) \times[0,3), \\
\{0\}, & \text { if } x=0,(s, t) \in[3,20] \times[3,20] .
\end{array}\right.
$$

By taking $f(t)=\cos t$ and $\varphi(t)=t^{4} /\left(1+t^{4}\right)$, for all $t \in[0$ ,20], then all the conditions of Theorem 21 are satisfied. Therefore, there exists at least one solution to the Fredholm integral inclusion:

$$
x(t) \in \cos t+\int_{0}^{20} L(t, s, x(s)) \sigma s, \quad t \in[0,20] .
$$

\section{Conclusion}

It is well-known that in some abstract spaces, the triangle inequality does not hold. But, by multiplying the constant $\eta$ $\geq 1$ on the right-hand side of the triangle inequality, one can obtain a more useful abstract structure, now called a $b$ -metric space in the literature. Following this improvement, in this work, an idea of $b$-hybrid multivalued contraction using the Hausdorff distance function is introduced in the setting of $b$-metric spaces. The established concept herein merges several results in one theorem. A few of these particular cases are stated. Thereafter, existence conditions for solutions of an integral inclusion of Fredholm type are discussed by applying one of the presented results herein.

While the presented results in this paper are theoretical, it is noteworthy that Hausdorff metric has numerous and useful applications in everyday life. For example, in computer vision, the Hausdorff distance can be applied to locate a given template in an arbitrary target image. The template and image are often preprocessed through an edge detector giving a binary image. Next, each one (activated) point in the binary image of the template is treated as a point in a set, the structure of the template. Similarly, an area of the binary target image is treated as a set of points. The algorithm then tries 
to minimize the Hausdorff distance between the template and some areas of the target image. The area in the target image with the minimal Hausdorff distance to the template can be taken as the best candidate for locating the template in the target. Moreover, in computer graphics, the Hausdorff metric is employed to measure the difference between two distinct representations of the same 3D objects, particularly when generating level of detail for efficient display of complex 3D models. For more applications of Hausdorff distance, see [34-36] and the references therein.

\section{Data Availability}

No data were used to support this study.

\section{Conflicts of Interest}

The authors declare that they have no competing interests.

\section{Acknowledgments}

This project was funded by the Deanship of Scientific Research (DSR), King Abdulaziz University, Jeddah, Saudi Arabia, under grant no. KEP-66-130-38. The authors, therefore, acknowledge with thanks DSR's technical and financial support.

\section{References}

[1] B. E. Rhoades, "A comparison of various definitions of contractive mappings," Transactions of the American Mathematical Society, vol. 226, pp. 257-290, 1977.

[2] S. B. Nadler, "Multi-valued contraction mappings," Pacific Journal of Mathematics, vol. 30, no. 2, pp. 475-488, 1969.

[3] H. Alsamir, M. S. M. Noorani, and W. Shatnawi, "Some fixed point theorems for $(\alpha, \psi, \varphi)$-contractive multivalued mappings in $b$-metric spaces," in AIP Conference Proceedings, vol. 1784, p. 050008, 2016.

[4] E. Ameer, H. Aydi, M. Arshad, H. Alsamir, and M. S. Noorani, "Hybrid multivalued type contraction Mappings in $\alpha_{K}$-complete partial $b$-metric spaces and applications," Symmetry, vol. 11, no. 1, p. 86, 2019.

[5] A. Azam and N. Mehmood, "Multivalued fixed point theorems in tvs-cone metric spaces," Fixed Point Theory and Applications, vol. 2013, no. 1, 2013.

[6] Q. Haitham, M. S. N. Noorani, W. Shatanawi, H. Aydi, and H. Alsamir, "Fixed point results for multi-valued contractions in $b$-metric spaces and an application," Mathematics, vol. 7, p. 132, 2019.

[7] M. A. Khamsi and N. Hussain, "KKM mappings in metric type spaces," Nonlinear Analysis: Theory, Methods and Applications, vol. 73, no. 9, pp. 3123-3129, 2010.

[8] M. Kutbi, E. Karapınar, J. Ahmad, and A. Azam, "Some fixed point results for multi-valued mappings in $b$-metric spaces," Journal of Inequalities and Applications, vol. 2014, no. 1, Article ID 126, 2014.

[9] H. K. Pathak, R. P. Agarwal, and Y. J. Cho, "Coincidence and fixed points for multi-valued mappings and its application to nonconvex integral inclusions," Journal of Computational and Applied Mathematics, vol. 283, pp. 201-217, 2015.
[10] I. A. Bakhtin, "The contraction mapping principle in almost metric spaces," Functional Analysis, vol. 30, pp. 26-37, 1989.

[11] N. Bourbaki, Topologie Generale, Herman, Paris, France, 1974.

[12] S. Czerwik, "Contraction mappings in b-metric spaces," Acta Mathematica Informatica Universitatis Ostraviensis, vol. 1, no. 1, pp. 5-11, 1993.

[13] N. Hussain and Z. D. Mitrovic, "On multi-valued weak quasicontractions in $b$-metric spaces," Journal of Nonlinear Sciences and Applications, vol. 10, no. 7, pp. 3815-3823, 2017.

[14] T. Kamran, M. Samreen, and Q. U. L. Ain, "A generalization of $b$-metric space and some fixed point theorems," Mathematics, vol. 5, no. 2, p. 19, 2017.

[15] K. P. R. Rao, P. R. Swamy, and J. R. Prasad, "A common fixed point theorem in complex valued $b$-metric spaces," Bulletin of Mathematics and Statistics research, vol. 1, no. 1, pp. 1-8, 2013.

[16] E. Karapinar, "A short survey on the recent fixed point results on b-metric spaceS," Constructive Mathematical Analysis, vol. 1, no. 1, pp. 15-44, 2018.

[17] E. Karapinar and A. Fulga, "New hybrid contractions on $b$ metric spaces," Mathematics, vol. 7, no. 7, p. 578, 2019.

[18] S. N. Mishra, S. L. Singh, and R. Talwar, "Nonlinear hybrid contractions on Menger and uniform spaces," Indian Journal of Pure and Applied Mathematics, vol. 25, pp. 1039-1052, 1994.

[19] S. A. Naimpally, S. L. Singh, and J. H. M. Whitfield, "Coincidence theorems for hybrid contractions," Mathematische Nachrichten, vol. 127, no. 1, pp. 177-180, 1986.

[20] H. K. Pathak, S. M. Kang, and Y. J. Cho, "Coincidence and fixed point theorems for nonlinear hybrid generalized contractions," Czechoslovak Mathematical Journal, vol. 48, no. 2, pp. 341-357, 1998.

[21] V. Popa, "Coincidence and fixed points theorems for noncontinuous hybrid contractions," In Nonlinear Analysis Forum, vol. 7, pp. 153-158, 2002.

[22] J. Appell, E. De Pascale, H. T. Nguyêñ, and P. P. Zabreŭko, "Nonlinear integral inclusions of Hammerstein type," Topological Methods in Nonlinear Analysis, vol. 5, no. 1, pp. 111124, 1995.

[23] J. P. Aubin and A. Cellina, Set-Valued Maps and Viability Theory in Differential Inclusions (Vol 264), Springer-Verlag, Berlin, 1984.

[24] T. Cardinali and N. Papageorgiou, "Hammerstein integral inclusions in reflexive Banach spaces," Proceedings of the American Mathematical Society, vol. 127, no. 1, pp. 95-104, 1999.

[25] R. Kannan and D. O'Regan, "A note on the solution set of integral inclusions," Journal of Integral Equations and Applications, vol. 12, no. 1, pp. 85-94, 2000.

[26] A. Sîntamarian, "Integral inclusions of Fredholm type relative to multivalued $\varphi$-contractions," Seminar on Fixed Point Theory Cluj-Napoca, vol. 3, pp. 361-368, 2002.

[27] V. Berinde, "Generalized contractions in quasimetric spaces," in Seminar on Fixed Point Theory, pp. 3-9, Babes-Bolyai University, Cluj-Napoca, Romania, 1993.

[28] N. Hussain, D. Doric, Z. Kadelburg, and S. Radenovic, "Suzuki-type fixed point results in metric type spaces," Fixed Point Theory and Applications, vol. 2012, no. 1, Article ID 126, 2012.

[29] M. Boriceanu, "Fixed point theory for multivalued generalized contraction on a set with two $b$-metrics," Studia Universitatis Babes-Bolyai, Mathematica, vol. 4, no. 3, pp. 126-132, 2009. 
[30] I. A. Rus, Generalized contractions and applications, Cluj University Press, 2001.

[31] V. Berinde, Contractii Generalizate si Aplicatii, Volume 2, Editura Cub Press, Baie Mare, Romania, 1997.

[32] S. L. Singh and B. Prasad, "Some coincidence theorems and stability of iterative procedures," Computers and Mathematics with Applications, vol. 55, no. 11, pp. 2512-2520, 2008.

[33] E. Michael, "A selection theorem," Proceedings of the American Mathematical Society, vol. 17, no. 6, pp. 1404-1406, 1966.

[34] A. Andreev and N. Kirov, "Word image matching based on Hausdorff distances," in 2009 10th International Conference on Document Analysis and Recognition, pp. 396-400, Barcelona, Spain, 2009.

[35] M. Beauchemin, K. P. Thomson, and G. Edwards, "On the Hausdorff distance used for the evaluation of segmentation results," Canadian Journal of Remote Sensing, vol. 24, no. 1, pp. 3-8, 2014.

[36] A. Pujol, J. J. Villanueva, and J. L. Alba, "A supervised modification of the Hausdorff distance for visual shape classification," International Journal of Pattern Recognition and Artificial Intelligence, vol. 16, no. 3, pp. 349-359, 2011. 